\title{
Clinicopathological Study on TB Lymphadenopathy Case
}

Mohammad Mahabubul Alam Chowdhury ${ }^{1 *}$ Pran Gopal Datta ${ }^{2}$

Abu Sahfi Ahmed Amin²

Khondakar Abdullah Al Mahmud ${ }^{1}$ Kawsar Sultana ${ }^{3}$

'Department of Otolaryngology and Head-Neck Surgery Institute of Applied Health Sciences (IAHS) Chattogram, Bangladesh.

${ }^{2}$ Department of Otolaryngology and Head-Neck Surgery Sheikh Mujib Medical University Dhaka, Bangladesh.

${ }^{3}$ Department of Community Medicine Chattagram Maa- O- Shishu Hospital Medical College Chattogram, Bangladesh.

\footnotetext{
*Correspondence to:

Dr. Mohammad Mahabubul Alam Chowdhury Associate Professor

Department of Otolaryngology and Head-Neck Surgery Institute of Applied Health Sciences (IAHS)

Chattogram, Bangladesh.

Mobile : +8801819320667

Email : mahbubulent@gmail.com
}

Date of Submission $\quad: \quad 10.02 .2021$

Date of Acceptance : 20.02 .2021

www.banglajol.info/index.php/CMOSHMCJ

\begin{abstract}
Background: Tuberculosis in the form of pulmonary and extrapulmonary TB is common in developing countries like Bangladesh. Among extrapulmonary TB, lymphnode $T B$ is still common in our setting. So, the objectives of the present study were to evaluate theclinicopathological findings among the lymphnode TB cases in our context.
\end{abstract}

Materials and methods: This observational study was done from January 2017 to December 2019 among 300 lymphnode TB cases in a tertiary care hospital during a three-year study period. Patients were selected after obtaining informed written consent and clinical evaluation. Then enlarged lymphnodes were evaluated by FNAC or histopathology. If lymphnode TB features were found then the patients were included in our study. Other findings like metastasis, lymphoma or pyogenic lymphadenitis were excluded from this study. After collection, data was recorded and analyzed by Microsoft Excel.

Results: Among 300 cases of TB lymphadenitis subjects were commonly within the age groups 11-20 years (Male 63 and female 51) and 21-30 years(male 45 and female 42) with amale: female ratioof 1.22:1. Regarding different clinical signs and symptoms, low grade fever was found in $21(7 \%)$ cases, local lymphnode pain and tenderness was found in 67 (22\%) and 63(21\%) of subjects. Discharging sinuses was present in 33(11\%) patients, 35(12\%) had history of weight loss, 39 (13\%) had anorexia and 42(14\%) had history of night sweats. Regarding locationof different lymph node areas, 125(41.67\%) patients had deep cervical nodes, 65(21.67\%) had supraclavicular lymph nodes, 60(20\%) had lymph nodes in the posterior triangle 33 (11\%) in jugulo-omohyoid and 17 (5.66\%) in submandibular area. Among all cases, 83\% cases showed bilateral and $17 \%$ showed unilateral involvement.Regarding nature of lymph nodes, firm, matted multiple lymphnodes were found in 130(43\%) cases, single discrete nodes were found in $70(23.33 \%)$ cases, others were found as suppurative noded with single and multiple sinuses. Cytopathological and histopathological findings revealed caseating granuloma with epithelioid cell with Langerhans type giant cell was found in $220(73 \%)$ cases, caseating granuloma with epithelioid cell was found in $47(16 \%)$ epithelioid cell was found in 20(7\%) cases and epitheliod cells with smear positive AFB was found in 13(4\%) cases.

Conclusion: TB lymphadenitis is still a common form of extrapulmonary tuberculosis and it has variable clinical and pathological presentations.

Key words: Lymph nodes; Tuberculosis; Clinicopathological.

\section{INTRODUCTIONS}

Tuberculosis (TB) is one of the most ancient diseases of humankind and it is prevailing in the society perhaps for several million years ${ }^{1}$. Tuberculosis is caused by a group of closely related bacterial species termed Mycobacterium tuberculosis complex. 
Today the principal cause of human tuberculosis is Mycobacterium tuberculosis. Other members of the $\mathrm{M}$. tuberculosis complex that can cause tuberculosis include M. bovis, M. microti and $\mathrm{M}$. africanum ${ }^{2}$.

Among the different lymphoid tissues lymph nodes are the most widely distributed and easily accessible component. Palpable lymph nodes are not always pathological but are presumed to reflect more frequent exposure to new pathogens or foreign bodies. The lymph nodes usually respond to a wide variety of stimuli. Lymphadenopathy is defined as an abnormality in size or character of the lymph node caused by invasion or propagation of either inflammatory cells or neoplastic cells into the node ${ }^{3}$. Lymphadenopathy is common clinical manifestation of broad categories of diseases like infections, malignancy and autoimmune disorders. Enlarged lymphadenopathy often presents a diagnostic dilemma. In countries like Bangladesh tubercular lymphadenoapthy comes at the top of the list. ${ }^{4}$ Aim of this study is to evaluate the climicopathological findings of lymphnode TB in our context.

\section{MATERIALS AND METHODS}

This was an observational study done in a tertiary care hospital in outdoor and indoor setting among the diagnosed lymphnode TB cases done by FNA Cand/or biopsy findings. Study period were three years from January 2017 to December 2019, Patients suspected on TB lympahadenitis clinically were undergone FNAC and/or biopsy after informed written consent and if TB diagnosis were confirmed were included in the study. Cytology of histopathology findings other than LNTB like malignancy, lymphoma or pyogenic lymphadenitis were excluded from the study. Clinical features were recorded and all data were complied and analyzed by Microsoft Excel.

\section{RESULTS}

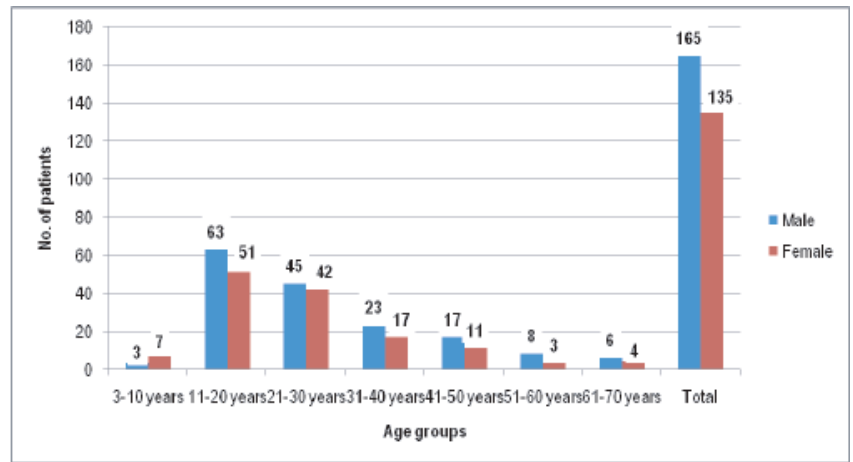

Figure 1: Distribution of patients according to age and gen$\operatorname{der}(n=300)$.

Figure 1 shows the gender and age group distributions of the 300 study patients of TB lymphadenitis where more patients were in the age group 11-20 year (Male 63 and female 51) and at age group 21-30 years (Male 45 and female 42). A total of $165(55 \%)$ males and $135(45 \%)$ females were in the study with a male to female ratio of $1.22: 1$.
Table I : Clinical signs and symptoms of the study patients $(n=300)$.

\begin{tabular}{lcc} 
Symptoms / Signs & Number & Percentage \\
Low grade fever & 21 & $7 \%$ \\
Local pain & 67 & $22 \%$ \\
Local Tenderness & 63 & $21 \%$ \\
Discharging sinus & 33 & $11 \%$ \\
Weight loss & 35 & $12 \%$ \\
Anorexia & 39 & $13 \%$ \\
Night sweats & 42 & $14 \%$ \\
\hline
\end{tabular}

Table I showing different clinical signs and symptoms where low grade fever was found in $21(7 \%)$ cases, local lymphnode pain and tenderness was found in $67(22 \%)$ and $63(21 \%)$. $33(11 \%)$ had discharging sinuses, $35(12 \%)$ had history of weight loss, 39 (13\%) had anorexia and $42(14 \%)$ had history of night sweats.

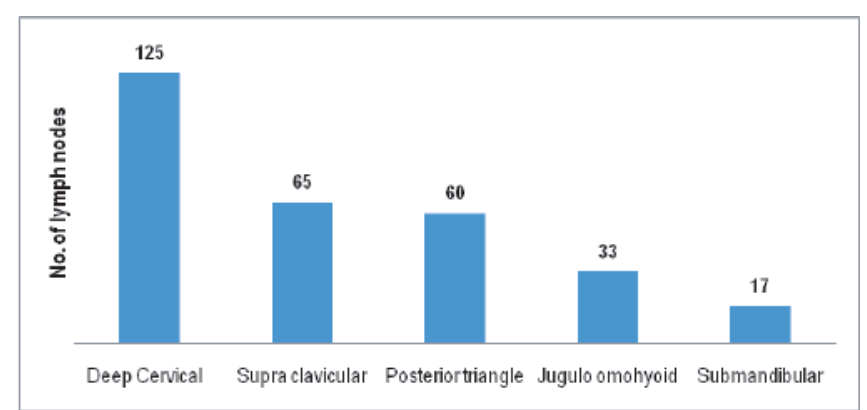

Figure 2 : Location of involved lymph nodes $(n=300)$.

Figure 2 displays the location of different lymph node areas where deep cervical nodes were $125(41.67 \%)$, supraclavicular lymph nodes were $65(21.67 \%)$, posterior triangle were $60(20 \%)$ others were found in jugulo-omohyoid and submandibular areas.

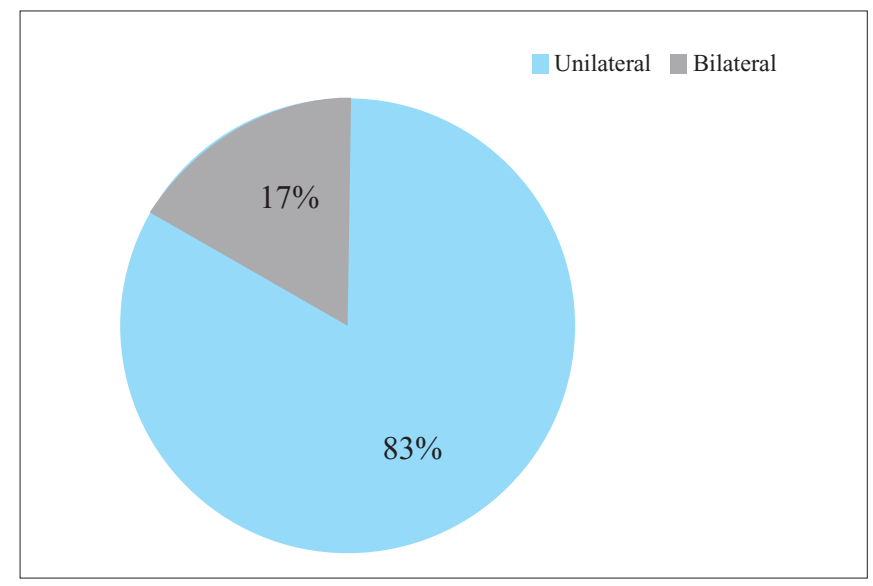

Figure 3 : Site of involvement $(\mathrm{n}=300)$.

The figure above shows the different sites of lymph node involvement. $83 \%$ cases had bilateral and $17 \%$ had unilateral lymph node involvement. 
Table II : Nature of involved lymph nodes $(\mathrm{n}=300)$.

\begin{tabular}{lcc} 
Nature & Number & Percentage (\%) \\
Firm, matted, multiple, lymph node & 130 & $43 \%$ \\
Single discrete & 70 & $23 \%$ \\
Suppurative lymph node & 75 & $25 \%$ \\
Discharging sinus & 25 & $9 \%$ \\
\hline
\end{tabular}

Table II shows the nature of lymph nodes among the study subjects. Firm, matted multiple lymphnodes were found in $130(43 \%)$ cases, single discrete nodes in $70(23 \%)$ cases, the restwere found to be suppurative lymph nodes with discharging sinuses.

Table III : Histopathological findings of lymphadenitis cases $(n=300)$.

Histopathological findings Number Percentage

Caseating Granuloma + Epitheloid cells +

Langerhan's type giant cell

Caseating Granuloma \& Epitheloid cells

Epitheloid cell

Epitheloid cells + Smear positive

Tubercular Bacilli

$\begin{array}{rr}220 & 73 \% \\ 47 & 16 \% \\ 20 & 7 \% \\ 13 & 4 \%\end{array}$

Table III shows thedifferent histopathological findings of the lymph nodes, where caseating granuloma with epithelioid cell with Langerhans type giant cell was found in 220(73\%) cases, caseating granuloma with epithelioid cell was found in $47(16 \%)$ epithelioid cell was found in $20(7 \%)$ cases and epithelioid cells with smear positive AFB was found in 13(4\%) cases.

\section{DISCUSSIONS}

Extrapulmonary tuberculosis affects lymph nodes, gastrointestinal tract, musculoskeletal system, genitourinary system, central nervous system, pleura, pericardium, although any organ can be involved. Hippocrates recognized the severity of abdominal TB by pointing out that diarrhoea attacking a person with emaciation is a mortal symptom. In Bangladesh, TB case notifications have increased significantly since 2012, mainly driven by increased numbers of extra-pulmonary and clinically diagnosed pulmonary cases ${ }^{5}$.

Among 300 cases, gender and age group distributions of TB lymphadenitisrevealed more patients were at age group 11-20 year (Male 63 and female 51) and at age group 21-30 years (Male 45 and female 42). We found most of the patients were at younger age group. Age differs between patient populations with LNTB and PTB. Farer et al. documented a skewed unimodal distribution towards younger age (25-34 years) in LNTB populations, whilst displaying bimodal distribution in their PTB population with peaks at 25-34 years and $65+$ years ${ }^{6}$.

In our study more case was in male $165(55 \%)$ and $135(45 \%)$ were female and male to female ratio was found 1.22:1. A study done by Biswas et al. ${ }^{5}$ found in their all cases of lymphnode TB female were affected more than the male patients. But a study done by Khandkar et al found female to male ratio for LNTB was 2.8:1, which is consistent with previous studies that found that LNTB is more common among women but contrast to our present study ${ }^{7}$. In Bangladesh most poor females works in different Garment factories, live in a crowded place and they are unaware of health hygiene. Other studies by Rajesh Kumar Padhy et al, Pandav et al, who found male preponderance with a male to female ratio being $1.17: 1^{8,9}$

Different clinical signs and symptoms revealed low grade fever was found in $21(7 \%)$ cases, local lymph node pain and tenderness was found in 67(22\%) and 63(21\%). 33(11\%) had discharging sinuses, $35(12 \%)$ had history of weight loss, $39(13 \%)$ had anorexia and $42(14 \%)$ had history of night sweats. These are some common constitutional presentation of extrapulmonary TB. General signs (Weight loss, sweats, fever, and asthenia) are found in 20 to $50 \%{ }^{10}$.

Different lymph node area analysis revealeddeep cervical nodes were $125(41.67 \%)$ supraclavicular lymph nodes were $65(21.67 \%)$ posterior triangle were $60(20 \%)$ others were found in jugulo-omohyoid and submandibular areas.Among all, 83\% cases were bilateral and $17 \%$ were unilateral involvement. Nature of lymph nodes revealed firm, matted multiple lymphnodes were found in 130(43\%) cases, single discrete nodes in $70(23 \%)$ cases, the rest were found to be suppurative lymph nodes with discharging sinuses. Lymphadenopathy has been defined as cervical lymph nodesmeasuring more than $1 \mathrm{~cm}$ in diameter. It is most frequentamong all age groups. Significant anxiety surrounds thefinding of cervical lymphadenopathy both to the patientand to the attending clinician, due to the concern ofthe underlying pathology. Numerous studies have beenconducted on cervical lymphadenopathy. Cervical lymphnodes are the most frequently enlarged and biopsied nodes, of all the peripheral lymph nodes ${ }^{11}$.

Histopathological findings where caseating granuloma with epithelioid cell with Langerhans type giant cell was found in $220(73 \%)$ cases, caseating granuloma with epithelioid cell was found in $47(16 \%)$ epithelioid cell was found in $20(7 \%)$ cases and epithelioid cells with smear positive AFB was found in $13(4 \%)$ cases. Lymph nodes are encapsulated centers of antigenpresentation and lymphocytic activation, differentiationand proliferation. They produce mature, antigen-primed, B and $\mathrm{T}$ cells and filter particles, including microbes, fromthe lymph by the action of numerous phagocytotic macrophages. These specialized immune cells named aslymphocytes, detect and combat the pathogens in thebody. When inflamed these nodes get swollen, enlarges toproduce the greater number of lymphocytes.

\section{CONCLUSION}

Tuberculous lymphadenitis represents about $10 \%$ of cases of tuberculosis in Bangladesh and is frequently the sole manifestation of extrapulmonary tuberculosis. Disease rates are highest among patients aged 21-30 years. Tuberculous lymphadenitis may respond slowly to standard antibiotic treatment, with persistent discomfort. Frequent patient follow-up during treatment is recommended for reassurance and management of local discomfort and further study is needed as an adjunct to standard antibiotic therapy to improve the otherwise slow response to treatment.

\section{DISCLOSURE}

All the authors declared no competing interest. 


\section{REFERENCES}

1. Hirsh AE, Tsolaki AG, DeRiemer K, Feldman MW, Small PM. Stable association between strains of Mycobacterium tuberculosis and their human host populations. Proc Natl Acad Sci USA. 2004;101:4871-4876.

2. Prasad H, Singhal A, Mishra A, Shah N, Katoch V, Thakral S et al. Bovine tuberculosis in India: Potential basis for zoonosis. Tuberculosis. 2005;85:421-428.

3. Benjelloun A, Darouassi Y, Zakaria Y, Bouchentouf R, Errami N. Lymph nodes tuberculosis: a retrospective study on clinical and therapeutic features. Pan Afr Med J. 2015; 20: 65.

4. National Tuberculosis Control Programme (NTP). Tuberculosis control in Bangladesh. Annual Report 2015. Dhaka: Director General of Health Services. 2015.

5. Biswas RRS, Chowdhury MFK, Mamun SMH. Clinicopathological profile and outcome of 29 lymph node tuberculosis cases. J Sci Soc. 2018;45:76-79.

6. Golden P M, Vikram R H. Extrapulmonary tuberculosis: An overview. Am Fam Physician. 2005;72(9):1761-1768.

7. Khandkar C, Harrington Z, Jelfs PJ,Sintchenko V, Dobler CC Epidemiology of Peripheral Lymph Node Tuberculosis and Genotyping of M. tuberculosis Strains: A Case-Control Study PLoS One. 2015; 10(7): e0132400.

8. Padhy RK, Maheswari A, Kumar Das B. A clinico pathological study of cervical lymphadenopathy. JEMDS. 2015;504:3497-3507.

9. Pandav AB, Patil PP, Lanjewar DN. Cervical lymphadenopathy - diagnosis by FNAC. A study of 219 cases. Asian J Med Res. 2012;1:79-83.

10. Hochedez P, Zeller V, Truffot $\mathrm{C}$ et al. Lymph-node tuberculosis in patients infected or not with HIV: General characteristics, clinical presentation, microbiological diagnosis and treatment. Pathologie Biologie. 2003;51(8-9):496-502.

11. Pandit AA, Candes FP, Khubchandani SR. Fine needle aspiration cytology of lymph nodes. J Postgrad Med. 1987;33:134-136. 